\title{
Performances of multi-PMT photodetector for the Hyper-Kamiokande experiment
}

\author{
Lucas Nascimento Machado*t \\ University of Naples Federico II - INFN Naples \\ E-mail: lucas.machado@na.infn.it
}

\begin{abstract}
Hyper-Kamiokande, a 187 kton fiducial volume water Cherenkov detector to be built in Japan, is the next generation of the Super-Kamiokande experiment. Its broad physics program includes nucleon decay, neutrinos from astronomical and human-made beam, with the main focus to determine the leptonic CP violation. To detect the weak Cherenkov light generated by neutrino interactions or proton decay, the primary photo-detector candidate are 20-inch PMTs. In order to enhance the Hyper-Kamiokande physics sensitivity, the use of multi-PMT modules is considered as a complement of the primary candidates. A multi-PMT Optical Module based on a pressure vessel instrumented with multiple small diameter photosensors, readout electronics and power, offers several advantages as increased granularity, reduced dark rate, weaker sensitivity to Earth's magnetic field, improved timing resolution and directional information with an almost isotropic field of view. Here we will present the recent development of the mechanical design and electronics of these modules in order to both maximize the physics sensitivity of Hyper-Kamiokande and comply with the detector requirements. We will then show the exhaustive tests of the individual 3-inch PMTs that constitute these modules before to present the incoming perspectives of testing the assembled modules in a test beam.
\end{abstract}

European Physical Society Conference on High Energy Physics - EPS-HEP2019 -

10-17 July, 2019

Ghent, Belgium

\footnotetext{
* Speaker.

${ }^{\dagger}$ On behalf of the Hyper-Kamiokande Proto-Collaboration.
} 


\section{Hyper-Kamiokande}

Hyper-Kamiokande (HK) is the next generation Water-Cherenkov detector with multi-purpose scientific goals, as the investigation on CP-violation in leptonic sector, determination of the neutrino mass ordering, observation of Cosmic Neutrinos and Proton Decay.

The detector will be a cylindrical tank with 71 meter height and 68 meters diameter. The total and fiducial masses will be respectively $260 \mathrm{ktons}$ and $187 \mathrm{ktons}$, which are about ten times larger than Super-Kamiokande.

The construction of the tank is planned to start in April, 2020 and the experiment will be ready for data taking in 2027. There are proposals for the construction of a second tank in Korea.

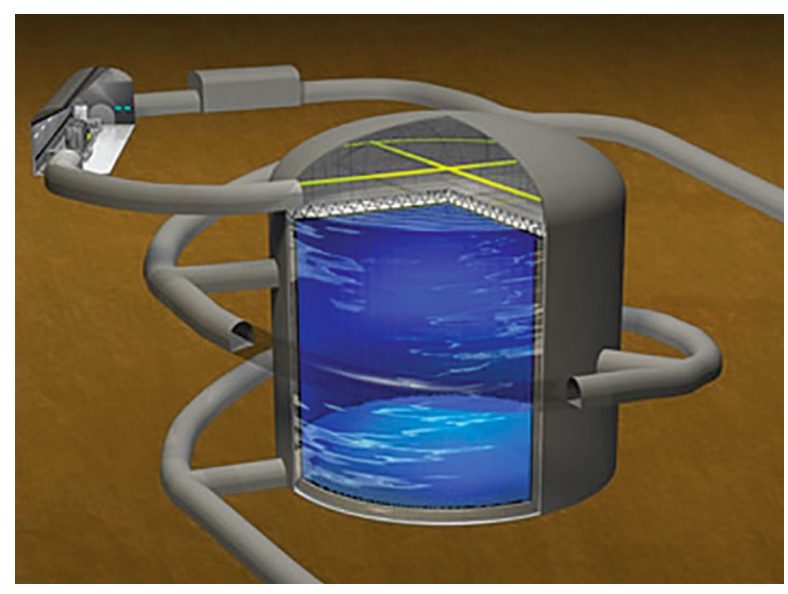

Figure 1: Illustrated scheme of the Hyper-Kamiokande Detector

Accelerator neutrinos measurement from the complex JPARC will be also improved with a higher intensity beam. A new water Cherenkov detector of $1 \mathrm{kton}$ at about $1 \mathrm{~km}$ from the source, the Intermediate Water Cherenkov Detector (IWCD), will provide flux predictions in HK and reduce systematic errors related to neutrino cross section uncertainty.

HK will also have enhanced photodetectors with improved performances, as a new geometry, better timing resolution and detector efficiency [1, 2]. The baseline design foresees 40,000 20-inch photomultiplier tubes (PMTs) observing the Inner Detector, and 6700 PMTs in the Outer Detector, which acts as veto for the entering particles.

Following requirements for the HK detector, alternative designs have been studied in order to improve the experiment physics capabilities. One of them, the multi-PMT (mPMT) concept, is presented next.

\section{The mPMT concept}

The mPMT module is an array of small photodetectors and their electronics arranged inside a pressure resistant vessel. It was first implemented in the KM3NeT experiment [3], which showed advantages like:

- Improved segmentation: Single-photon hits can be distinguished from multi-photon hits analysing the signal in neighboring PMTs. 
- Superior photon counting: Total number of deposited photoelectrons can be directly obtained from hits on PMTs.

- Local coincidences: When considering coincidences between invidual PMT inside the modules, suppression of uncorrelated single-hit noise can be improved.

- Directional sensitivity: With the individual direction of each single PMT, the mPMT gives information on the direction of detected photons, improving signal-to-background separation.

- Extended dynamic range: When multiple photons arrive at the same time, it will be more likely to hit different PMTs.

An alternative option for HK is to have a combination of the 20-inch PMTs and the mPMTs modules. The IWCD will be based on the mPMT concept.

\section{1 mPMT Geometry for Hyper-Kamiokande}

As illustrated in Figure 2, the basic geometry of the mPMT module for HK is a cylindrical vessel with a approximate $26 \mathrm{~cm}$ radius, housing 19 3-inch PMTs. The PMTs are supported by a 3D printed structure and optically coupled by Silicon Gel. The vessel has an acrylic cover and metallic body.

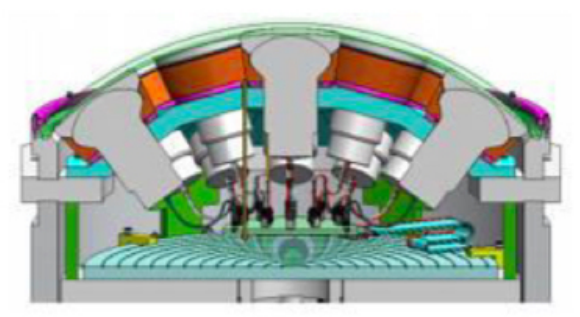

Figure 2: mPMT design

The front-end board is integrated inside the vessel, as well as the HV supplier for the PMTs. To each small photodetector is added a reflector cone, in order to increase the effectiveness of the photocathode area.

The modules will be facing the Inner Detector, increasing its granularity.

\section{2 mPMT Prototypes for Hyper-Kamiokande}

Designs of mPMT modules for the HK experiment have been in development, taking into account different configurations for the PMTs, vessel materials, PMT read-outs, assembling processes, etc.

The initial prototypes, which are under test, are:

- INFN Prototype: based on KM3NeT experiment, a mPMT module with a 17-inch vessel and 3-inch PMTs inside to test the acrylic vessel and electronics.

- TRIUMF Prototype: optimized design to test of new mechanics and assembling procedure. 

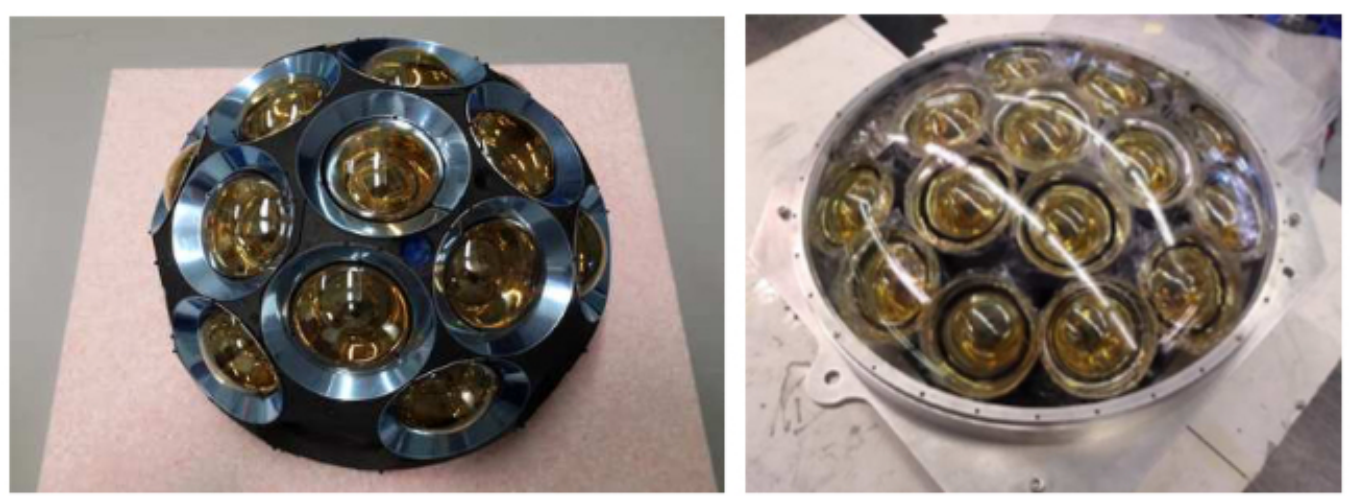

Figure 3: The two initial prototypes for Hyper-Kamiokande from INFN (left) and TRIUMF (right)

Preliminary simulation studies have already showed improved vertex resolution and increased PID separation compared to 20-inch PMTs [4].

\subsection{Acrylic Vessel}

$\mathrm{Km} 3 \mathrm{NeT}$ experience demonstrated that glass vessels are characterized by high ${ }^{40} \mathrm{~K}$ and other radioactive contamination. Tests on acrylic samples from several companies have been performed to identify the best solution for HK.

Tests of optical and mechanical performances, absorption, compatibility with optical gel, radioactive contamination, and pressure resistance, showed that the 'PLEXIGLAS GS UV transmitting' by Evonik is the best solution for the mPMT module. Results of optical transmittance test in different acrylic materials are in Figure 4. A hydrostatic pressure test has been realized for $15 \mathrm{~mm}$ and $20 \mathrm{~mm}$-thick spherical vessels to verify the safe constraint to resist to $1.26 \mathrm{MPa}$. No damage has been registered up to $1.8 \mathrm{MPa}$ for both the vessels, much higher than the HK constraints.

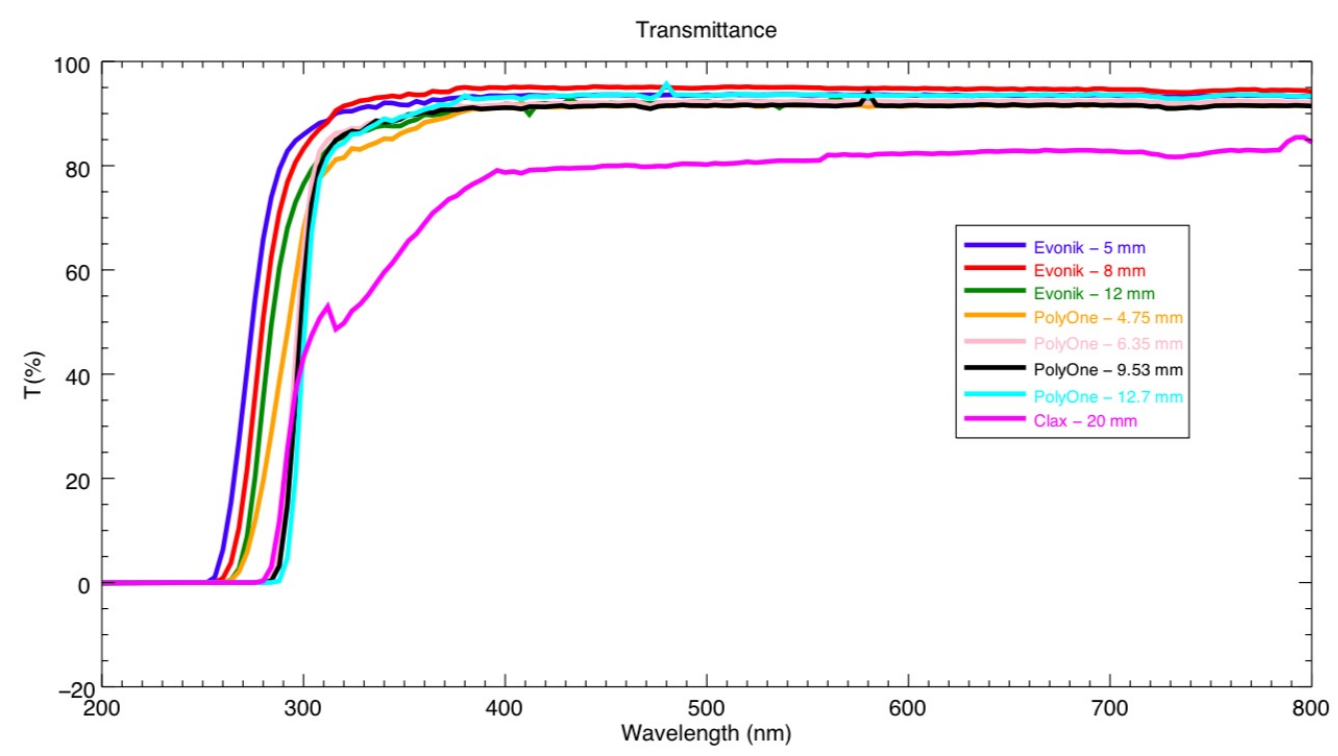

Figure 4: Acrylic samples transmittance 
Nuclear tests at the National Gran Sasso Laboratories (LNGS) have been realized to investigate radioactive contaminations by measuring gamma emissions, showing radioactivity levels comparable to the 'KURARAY PARAGLAS-UV00' acrylic curretly used in Super-Kamiokande [5]

\section{4 mPMT electronics}

Following the requirements for HK, the development of the electronics for the mPMT modules needs to take into account low power consumption and good charge and time resolution.

The HV supplier is a basic Cockcroft-Walton voltage multiplier circuit, used to generate multiple voltages for the PMT. It raws less than $1.5 \mathrm{~mA}$ of supply current at $3.3 \mathrm{~V}$ and can provide up to $-1500 \mathrm{~V}$ cathode voltage.

Two different designs for the mPMT digitization are in development: a Q/T digitization based on discrete components and one based on FADC digitization, with on-board signal processing.

Current prototypes of both the PMT base and the digitizer unit based on Q/T digitization with a TDC and a 2-step integrator coupled to a sample-and-hold ADC showed good performance. The achieved timing resolution is $\sim 100 \mathrm{ps}$, the charge resolution is $0.1 \%$ and good linearity of the circuit response to charge was observed.

The system has very low power consumption, reaching $12.5 \mathrm{~mW}$ for the HV circuit and 40.5 $\mathrm{mW}$ for the digitizer per-channel, with a total power consuption of $\sim 4 \mathrm{~W}$ per mPMT module [5].

\section{Summary}

The alternative option of a mPMT system for the HK detector design will offer many benefits to experiment. Some the advantages of the mPMT concept are improved segmentation, superior photon counting, directional sensibility and increase of granularity.

The two mPMT prototypes from INFN and TRIUMF are currently under test and the results will point the optimizations needed to make the final design for mPMT option for the HK experiment.

\section{References}

[1] Hyper-Kamiokande Proto-Collaboration, Hyper-Kamiokande Design Report, [physics.ins-det/1805.04163].

[2] Y. Nishimura, on behalf of the Hyper-Kamiokande Proto-collaboration, New 50-cm Photo-Detectors for Hyper-Kamiokande in proceedings of International Conference on High Energy Physics, POS (ICHEP 2016) (2017).

[3] Adrian-Martinez, et al. (KM3NeT Collaboration), Deep sea tests of a prototype of the KM3NeT digital optical module, Eur. Phys. J. C74 (9) (2014) 3056 [astro-ph/1405. 0839].

[4] B. Quilain, Multi-PMT modules for Hyper-Kamiokande, Proceedings in preparation, [https://www-conf.kek.jp/PD18].

[5] Gianfranca De Rosa, on behalf of Hyper-Kamiokande Proto-Collaboration, A multi-PMT photodetector system for the Hyper-Kamiokande experiment, NIMA PROCEEDINGS-D-19-00151, [DOI:10.1016/j.nima.2019.163033]. 